\title{
Détermination de la densité normale du gaz éthylène
}

\author{
par \\ T. Batuecas. \\ (25. IV. 1918.)
}

Les données concernant la densité exacte du gaz éthylène sont très rares. Quelques déterminations anciennes, entachées de toutes les causes d'erreur de l'époque antérieure à Regnault, n'ont qu'une valeur historique; depuis lors, la densité de l'éthylène a été déterminée par Bretschger ${ }^{1}$ ) sur un gaz provenant de la réduction du bromure d'éthylène par le zinc, préalablement soumis à une simple purification chimique, et par Stahrfoss ${ }^{2}$ ) à Genève, avec un gaz purifié par distillation fractionnée suivant la technique moderne. De tous ces résultats, ceux de Stahrtoss sont seuls à retenir; cependant les mesures de cet observateur sont peu nombreuses et ont été exécutées avec du gaz éthylène d'une seule provenance; elles paraissent affectées d'une petite erreur systématique du fait du baromètre utilisé, question qui doit être encore examinée à Genève.

En raison de ces considérations, et sur le conseil de M. le Prof. Ph.-A. Gutye, nous avons entrepris la révision de la densité du gaz éthylène. La marche suivie pour ces recherches a été celle mise au point à Genève depuis quelques années, avec les perfectionnements apportés par $E$. Moles dans un travail récent sur la densité du gaz bromhydrique ${ }^{3}$.

Préparation du gaz éthylène. Presque toutes les méthodes pratiques de préparation sont basées sur la déshydratation de l'alcool par les acides, certains sels et oxydes. Nous avons obtenu ce gaz par les méthodes suivantes:

I. Déshydratation par l'acide phosphorique sirupeux

II. Déshydratation par l'acide borique anhydre

III. Déshydratation par l'acide sulfurique concentré

IV. Déshydratation catalytique par l'action de l'alumine.

1) Bretschger, Dissert. Zurich, 1911.

2) Stahrfoss, Thèse, Genève, 1914.

3) E. Moles, Thèse, Genève 1916, J. Ch. phys. 14, 389 (1916). 
La première méthode a déjà été mise au point par Stahrfoss. Nous nous bornerons à indiquer qu'avec l'emploi de l'acide sirupeux, il suffit de chauffer le mélange dans un bain de limaille de fer un moment à $200^{\circ}$. et ensuite à $150-160^{\circ}$ (Stahrfoss indique $220-230^{\circ}$ ). Le dégagement est assez régulier; nous avons remarqué qu'à une température plus haute, il se produit un boursouflement de la masse qui devient brunâtre et que l'acide cesse d'agir au bout d'un certain temps. Les gaz traversent une solution de potasse caustique $(1: 1)$, un condenseur $\left(\grave{d}-80^{\circ}\right)$ pour retenir l'excès d'alcool et l'éther, un tube contenant de la potasse caustique solide, un laveur à acide sulfurique et un tube à anhydride phosphorique, après quoi il est condensé et fractionné, avec barbotage dans le gaz liquéfié.

La seconde méthode que l'on considère généralement comme donnant un gaz très pur, présente des difficultés pratiques très grandes. Il se produit dans la réaction entre l'alcool et l'acide borique anhydre de l'éther éthylborique qui, décomposé par la chaleur, donne de l'éthylène et de l'acide borique hydraté. Ce dernier forme des dépôts très consistants sur les parois de l'appareil à dégagement, dont l'orifice de sortie est facilement obstrué. D'autre part, le rendement très médiocre oblige à chauffer vers $300^{\circ}$, ce qui cause des accidents dûs à l'attaque rapide des ballons de verre par l'acide borique. Le gaz ainsi produit a été lavé à l'eau et avec une solution de potasse caustique; il passe ensuite sur de la potasse caustique solide et de l'anhydride phosphorique; il a été employé directement, étant donné que le faible volume obtenu ne permettait pas le fractionnement.

La troisième méthode a été pratiquée suivant la variante indiquée par Cardoso et $\left.A r n i^{1}\right)$. On chauffe un mélange d'alcool et d'acide sulfurique $(1: 2)$ additionné d'un peu de sable lavé et calciné. Le dégagement a été abondant et très régulier vers 140 à $150^{\circ}$; il ne se forme pas de mousse. Le gaz purifié par son passage dans la potasse caustique (solution), l'acide sulfurique concentré et l'anhydride phosphorique est ensuite condensé avec l'air liquide et fractionné avec barbotage.

La quatrième méthode a exigé quelques essais préalables. La variante adoptée définitivement consiste à faire passer la vapeur d'alcool dans un tube de verre de Bohême contenant de l'alumine

1) Cardoso et Arni, J. Cls. phys. 10, 504 (1912). 
commerciale (préalablement calcinée deux fois au rouge sombre), et chauffée à $350-370^{\circ}$ dans une gouttière garnie de limaille de fer. Le dégagement est très régulier et très rapide et le gaz débarrassé d'eau et de l'excès d'alcool dans deux condenseurs refroidis par le mélange carbonique $\left(-80^{\circ}\right)$ a été liquéfié et fractionné avec barbotage sans subir aucune autre purification chimique.

D'après Senderens ${ }^{1}$ ), l'action catalytique de l'alumine est très différente suivant la nature du produit et le mode de calcination, tandis que le sulfate d'aluminium agit beaucoup plus sûrement et donne $99,5 \%$ d'éthylène. Nous avons donc rempli notre tube à moitié avec du sulfate d'aluminium (Kahlbaum) calciné suivant Senderens, et l'autre moitié avec de l'alumine qui devait agir comme substance desséchante ${ }^{2}$ ). Le dégagement gazeux a été considérable, mais la partie de gaz condensée était inférieure à $50 \%$, le reste étant sûrement de l'hydrogène. En outre, une grande quantité d'alcool, qui n'avait pas réagi, s'est condensée dans les canalisations. Nous avons attribué l'insuccès à la présence de l'alumine et avons rempli le tube une seconde fois entièrement avec le sulfate d'aluminium calciné. A notre étonnement, la quantité de gaz produit était presque nulle; l'alcool se condensait sans avoir réagi durant son passage sur le sulfate d'aluminium. Finalement, nous avons répété l'essai dans des conditions identiques, mais en employant de l'alumine calcinée exclusivement. Lie succès a été complet, le gaz dégagé a été presque totalement condensé par l'air liquide, tandis que le liquide retenu dans les condenseurs refroidis à la neige carbonique était de l'eau presque pure.

La seule différence entre notre mode expérimental et celui décrit par Senderens réside probablement dans le fait que nous avons opéré en faisant le vide dans l'appareil entièrement construit en verre soudé, et en maintenant, du fait de la condensation du gaz éthylène par le froid, une forte dépression dans cet appareil.

Le gaz destiné aux mesures a été purifié par fractionnement accompagné de barbotage dans l'éthylène liquide. Le barbotage a été répété deux ou trois fois. L'efficacité de ce mode de purification a été de nouveau constatée très nettement.

1) Senderens, A. Ch. [8] 25, 449 (1912).

2) Voir Johnson, Am. Soc. 34, 911 (1912). 
Il a déjà̀ été indiqué ailleurs que la valeur de la clensité du gaz éthylène est peu influencée par la présence de traces d'air comme impureté. D'après les méthodes de préparation adoptées, l'éthylène se dégage avec d'autres gaz, bioxyde de soufre, bioxyde de carbone, hydrogène et des vapeurs d'alcool, d'éther, d'eau, etc. Les impuretés produites par la déshydratation avec les acides tendraient, en général, à augmenter la densité du gaz éthylène, tandis que l'hydrogène qui se produit toujours par déshydratation catalytique influencerait la densité en sens contraire. Un coup d'oeil sur le tableau des résultats montre que le mode de purification a été très efficace.

La densité a été déterminée par la méthode des ballons, telle qu'on la pratique au Laboratoire de Chimie théorique de l'Université de Genève; elle a été décrite à plusieurs reprises et nous nous bornerons à relever ici que les lectures de pression et le dispositif manométrique étaient les mêmes que ceux employés par E. Moles (Loc. cit.). Quoique l'èthylène n'attaque pas le mercure, on a jugé très pratique l'emploi d'un manomètre compensateur à huile de paraffine qui sert comme indicateur d'équilibre de pression, surtout pendant les remplissages; les dénivellations du compensateur étant 15,5 fois plus grandes que celles $\mathrm{du}$ baromètre à mercure, on observe ainsi très rapidement les plus petites variations de pression.

Les ballons employés au nombre de trois avaient les capacités suivantes:

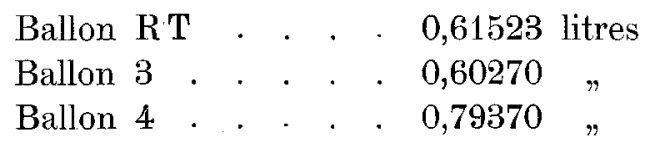

On a procédé par double pesée par substitution. La sensibilité de la balance était de $1 / 20 \mathrm{mg}$ par la méthode des oscillations.

Dans le calcul du poids du litre normal $\mathrm{L}_{\mathrm{o}}$ par la formule des gaz parfaits

$$
\mathrm{L}=\frac{\mathrm{S}}{\mathrm{C}} \cdot \frac{760}{\mathrm{P}_{\mathrm{o}}}
$$

il a été tenu compte des corrections provenant de la poussée de l'air sur les poids marqués, de la contraction des ballons, de la latitude et de l'altitude et de la compressibilité (coefficient de Leduc $\left.=103 \times 10^{-8}\right)$. 


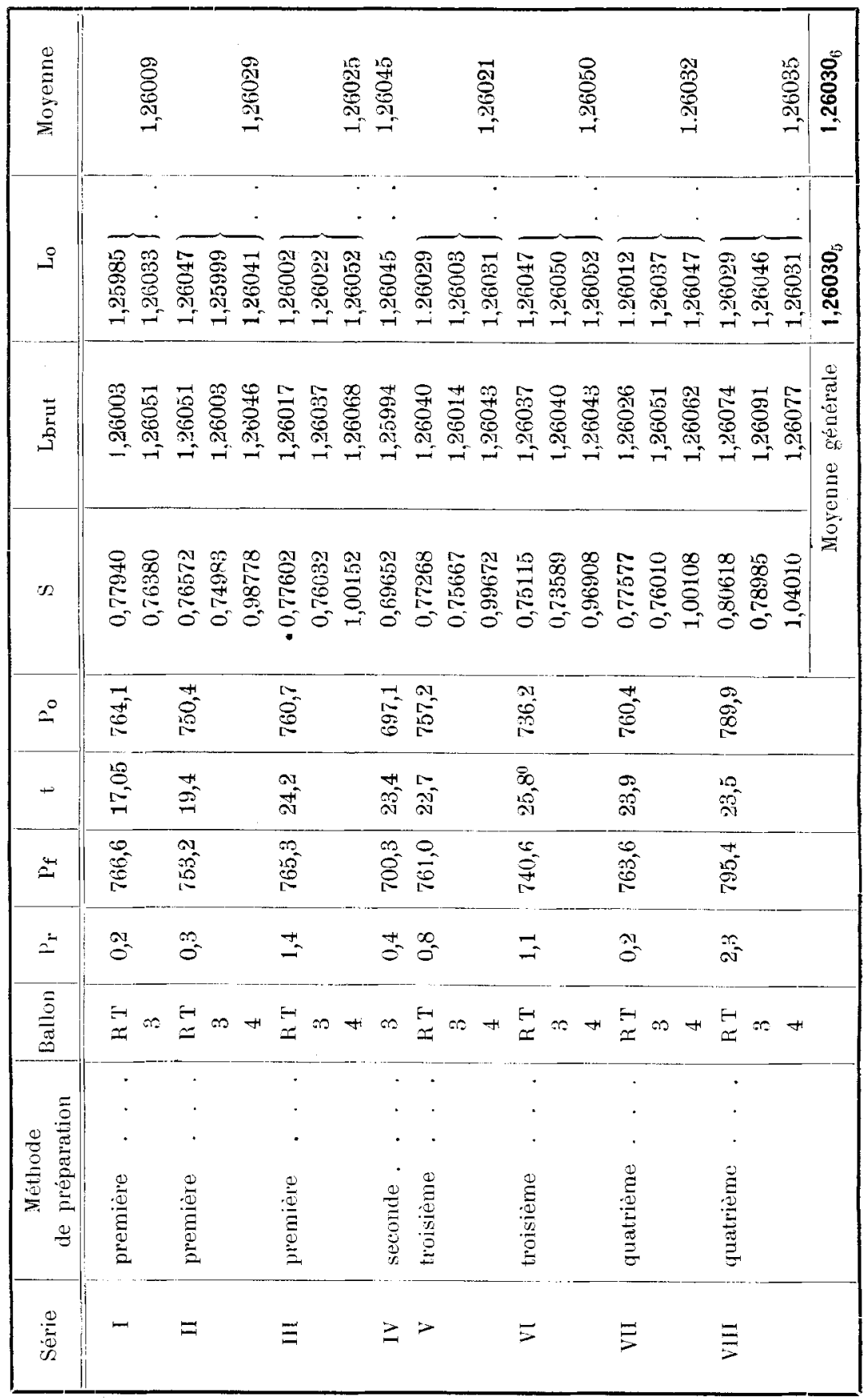


Dans le tableau suivant, on indique la pression résiduelle $\mathrm{P}_{r}$, la pression de fermeture $\mathrm{P}_{\mathrm{f}}$, celle de remplissage corrigée $\mathrm{P}_{o}$, le poids de substance $S$, le poids du litre brut $\mathrm{L}_{\text {brut }}$ et celui corrigé $\mathrm{L}_{0}$. Si l'on groupe les résultats par ballon, les moyennes sont:

\begin{tabular}{cc} 
Bailon & Moyenne \\
$\mathrm{R} \mathrm{T}$ & 1,26021 \\
3 & 1,26024 \\
4 & 1,26042 \\
\hline & $1,26030_{6}$
\end{tabular}

L'écart extrême pour les moyennes par série est de $\frac{\mathbf{3 , 1}}{\mathbf{1 0 , 0 0 0}}$ et de $\frac{1,6}{10,000}$ pour les moyennes par ballon. L'écart maximum sur la moyenne est de $\frac{\mathbf{3 , 6}}{\mathbf{1 0 , 0 0 0}}$ pour les déterminations isolées, $\frac{\mathbf{1 , 6}}{\mathbf{1 0 , 0 0 0}}$ pour les moyennes par série, $\frac{\mathbf{0 , 9 5}}{\mathbf{1 0 , 0 0 0}}$ pour les moyennes par ballon.

Le poids du litre normal de gaz éthylène, arrondi à la quatrième décimale, est donc:

$$
\mathrm{L}_{\mathrm{o}}=1,2603 \text {. }
$$

Genève, Laboratoire de Chimie théorique de l'Université, juillet 1917.

Les expériences résumées dans la présente note feront l'objet d'un mémoire détaillé dans le Journal de Chimie physique.

\section{Sur l'ionisation des gaz pendant les réactions chimiques}

par

\section{Alexandre Pinkus.}

(25. IV. 18.)

L'ionisation des gaz par réaction chimique a été pendant longtemps considérée par la plupart des physiciens comme un fait démontré, pouvant d'ailleurs trouver une explication dans la théorie électronique de la matière ${ }^{1}$ ). Cette manière de voir a suscité ces

1) Beattie. Ph. Mag. [5] 48, 97 (1899); [6] 1, 442 (1901). - G: Lebon. C. R. 130, 895 (1900). - Garret et Willows. Ph. Mag. [6] 8, 437 (1904). - J. Cunningham et C. Mukerji. Jahrb. Elektr. 4, 370 (1908). - G. Réboul. C. R. 149, 112 (1909). - Haber et Just. W. [4] 30,411 (1909) et Z. El. Ch. 16, 275 (1910); 17, 592 (1911); 20, 483 (1914). - Tanatar et Bursker. अR 45; 1 (1913); 47, 956 (1915) etc. 